\title{
Adaptive Navigation for Autonomous Robots
}

\author{
Matt Knudson ${ }^{\mathrm{a}}$, Kagan Tumer ${ }^{\mathrm{b}}$ \\ aknudsonm@engr.orst.edu \\ ${ }^{b}$ kagan.tumer@oregonstate.edu \\ Oregon State University \\ 204 Rogers Hall, Corvallis, OR USA 97331
}

\begin{abstract}
In many robotic exploration missions, robots have to learn specific policies that allow them to: (i) select high level goals (e.g., identify specific destinations), (ii) navigate (reach those destinations), (iii) and adapt to their environment (e.g., modify their behavior based on changing environmental conditions). Furthermore, those policies must be robust to signal noise or unexpected situations, scalable to more complex environments, and account for the physical limitations of the robots (e.g., limited battery power and computational power).

In this paper we evaluate reactive and learning navigation algorithms for exploration robots that must avoid obstacles and reach specific destinations in limited time and with limited observations. Our results show that neuroevolutionary algorithms with well-designed evaluation functions can produce up to $50 \%$ better performance than reactive algorithms in complex domains where the robot goals are to select paths that lead to seek specific destinations while avoiding obstacles, particularly when facing significant sensor and actuator signal noise.
\end{abstract}

Key words: Learning::Single Agent, Learning::Evolution, Learning::Neural Networks, Robotics::Adaptation

\section{Introduction}

Advances in mobile autonomous robots have provided solutions to complex tasks previously only considered achievable by humans. Such domains include planetary or underwater exploration $[1,2]$, operation in urban environments [3] and unmanned flight [4]. In each of those domains, autonomous 
navigation plays a key role in the success of the robots. However, as navigation has become more complex, algorithms have become both domain specific and resource intensive [5].

Indeed, successful navigation algorithms need to operate in partially observable and dynamic environments that are often stochastic in nature. One approach to providing such capabilities is the use of domain knowledge at the expense of high sensing, computational and power requirements [6]. Another approach is to provide a mapping from sensory inputs to actions that statistically capture the key behavioral objectives without needing a model or detailed domain knowledge [7]. Such methods are well-suited to domains where the tools available to learn from past experience and adapt to emergent conditions are limited.

In this work we explore one such approach; neuro-evolutionary based navigation [8]. Neuro-evolutionary approaches are policy search methods where control is achieved through a search across policies. This search through a population of policies allows for the discovery of new and robust control strategies. Neuro-evolutionary approaches have been successfully applied to both benchmark problems [9] and real world control problems [10, 11]. Often the policy, an artificial neural network, is simple in construction and therefore is inexpensive to modify and evaluate in practice, providing resource cost benefits as well $[12,13]$.

In this paper we provide a neuro-evolutionary policy search approach to adaptive navigation for robots with limited resources, operating in a partially observable environment. In addition, we explore the applicability of a learned policy from physics-based simulation to a physical robot. Section 2 describes the state and actions spaces, as well as the rule-based and neuro-evolutionary algorithms. Section 3 presents the problem domain as well as the robot capabilities. Section 4 presents the experimental approach, the hypothesis and the simulation results, where Section 5 presents hardware implementation results. Finally, Section 6 provides a summary and a discussion of the results as well as directions for further research.

\subsection{Contributions of this Work}

This work addresses learning adaptive navigation policies for robots with limited resources. Specifically, we developed unique state and action spaces on which the learning algorithm and a rule-based algorithm operated. In addition, we analyzed the applicability of control policies learned in simulation to a physical robot. More specifically our contributions are to: 
1. Develop State and Action Spaces: The learning algorithms have access to limited state information consisting of a distance to a potential obstacle and a calculated heading toward some destination. This information is provided for each of 360 possible paths for robot travel, one for each degree of possible movement direction. Rather than choosing a robot heading, the learning algorithms rank each of these paths (assign "quality") and the path with greatest quality is chosen for the robot heading. This state action space (as opposed to actions corresponding to specific robot headings) is domain independent and generalizable to many robotic applications.

2. Provide Neuro-evolutionary Navigation: An evolutionary algorithm is used to search through the parameter space of a neural network which assigns path qualities to specific directions. The neural network is run for each of the 360 potential paths at each time-step to establish a path quality distribution from which to choose the path of greatest quality. The robot heading is then assigned to the path with the highest quality. ${ }^{1}$

3. Applicability of Learned Policies: The simulator used for training the adaptive algorithm is physics-based and accurately represents the movement and capabilities of a simple differential-steering robot platform. However, to facilitate tractable computation speeds, certain aspects of real-world conditions were intentionally excluded. For all training applications this situation is likely to be the case, so we examined the behavior of policies learned in simulation when installed on an actual robotic platform, similar in construction.

The work presented in this paper develops this state/action space selection, provides a learning algorithm for navigation and analyzes the behavior of the algorithm in the real world.

\subsection{Related Work}

Establishing successful navigation policies for continuous and complex environments is an important challenge, particularly when the environment can only be partially observed by the robot. This can be accomplished in

\footnotetext{
${ }^{1}$ There is no exploration in action space here, because the exploration occurs at the policy level.
} 
a variety of ways, including using physical models of the robot and the environment in which it operates to determine the limits of functionality and interaction [14]. In general, planning techniques utilize such models as well, and have also proven successful for navigation in unknown environments and control of spacecraft $[15,16,17]$. Successful heuristic search based planning techniques include an algorithm Dynamic $A^{*}[18], \mathrm{D}^{*}$ heuristic planning [19], and a modified $\mathrm{D}^{*}$ replanning algorithm [20], which all utilize heuristic mechanisms for path planning. These techniques, while not exclusively requiring complete models, do however rely on deterministic sensing and action outcomes and therefore can require large processing capabilities. To alleviate this, the utilization of Markov models and adaptive techniques for use in sensor interpretation have been shown to improve navigation performance $[21,22]$ by injecting some flexibility into what the robot observes and how interpretation is achieved.

However, generating complete or even good models of an environment, either offline or online, can prove quite difficult. Therefore, navigation policies that effectively retain the ability to react to inaccurately generated or nonexistent environment models provide advantages [23, 24, 25, 26]. Specifically, adaptive techniques for tasks including rocket and helicopter control, walking robots, pole balancing, and underwater robotics have produced good results $[27,28,29,30,31]$. These techniques focus on control policies that either do not retain any specific models of their environment, or provide flexible models that adapt to changes to their environment.

Model-free learning algorithms such as reinforcement learning can also be used for navigation applications [32]. Various types of reinforcement learning have also been successfully applied to free gait development [33], dynamic job scheduling [34], and objective recognition for computer vision [35]. The online reinforcement learning algorithm OLPOMDP [36] has been used successfully in applications including autonomous underwater vehicles, general robot control, and vulnerability assessment in $\mathrm{P} 2 \mathrm{P}$ networks [37, 38, 39]. By operating on a parameterized functional representation of the knowledge gained during operation, instead of on specific models, important features of the world in which the robot (or agent) operates can be the focus.

\section{Robot Navigation}

In cases where resources are strongly limited, particularly in ability to observe the world surrounding the robot, the interpretation of incoming in- 
formation as it relates to actions to be taken is a very important area for attention. Therefore, the specifics of such robot navigation through unknown, complex environments need to be addressed. The robot must have the ability to choose safe and efficient paths through an environment to reach a specific destination. This includes the ability to avoid obstacles and maximize robot speed, while maintaining a level of robustness to inaccuracies (noise) in sensor and actuator signals.

In addressing these requirements, it is important to determine the benefits and drawbacks of a rule-based algorithm (i.e., does not modify behavior) as it compares to algorithms with the ability to learn and change behavior as they operate within an environment. This adaptive ability is important when the exact solution to effective navigation is unknown to the system designer as well as for situations where the algorithm can not be easily shut down and redesigned.

For this work, navigation through the environment was achieved using two techniques for controlling the robot based on environment information obtained from sonar and inertial sensors;

- Rule-Based Navigation: An algorithm is used to provide a rulebased action choice based on state information collected. The choice is based on predetermined probability distributions developed from known robot capabilities and empirical behavior data.

- Neuro-Evolved Navigation: This is a Multi Layer Perceptron (MLP) $[40,41,42]$ based controller designed under the same premise as the rule-based driver in that it interprets environment detection information and assigns a path quality to each potential path. However, it deviates in the interpretation method where the sensor information for that potential path is fed directly to an MLP and the output of the MLP is used as the path quality.

A random technique is used to produce a baseline, where the action taken at each time step is random. The navigation algorithms are discussed shortly, however it is beneficial to first discuss and understand the unique state and action spaces defined to account for the limited resources available to the algorithms.

\subsection{State and Action Spaces}

In the work presented here, the mobile robotic platform has a limited set of sensing capabilities (discussed in further detail in Section 3.1) as well as 
provides a non-deterministic outcome of actions taken. As a result, a unique set of spaces is required to accurately represent the environment surrounding the robot and provide maximum articulation capabilities.

To encode as much information as possible from the sensors, as simply as possible, incoming state details are distilled into two state variables:

- Object Distance: A distance to the nearest object $d_{\theta_{i}}$ is provided for each vehicle relative angle $\theta_{i}$. This represents a potential obstacle, such as a wall or rock.

- Destination Heading: The difference between the potential path heading $\theta_{i}$ and the vehicle relative destination heading $\alpha_{\text {des }}$ is provided. This indicates how significant of correction is required for the robot to point directly toward the destination.

This state representation is of course quite predictable in the destination heading, never exceeding $|180|$ degrees and symmetrical about the destination heading $\alpha_{\text {des }}$. The object distance state variable can vary widely however, and depends strongly on the resolution of the environment sensing available. Both state variables also depend on the accuracy of the sensors that provide distance and track robot orientation.

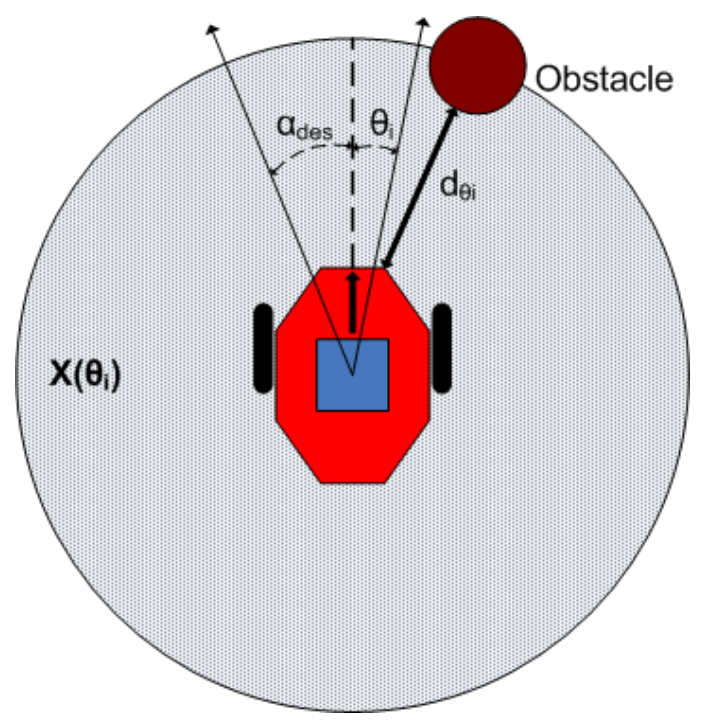

Figure 1: Graphical depiction of state and action spaces. 
Physical robots in general provide non-deterministic outcomes of actions requested. By and large this is due to the unpredictable environment in which the robot operates. In the mobile robot case, the interface between actuating devices (i.e., motors) and articulation devices (i.e., gears and wheels) as well as the interface between these devices and the environment (i.e., terrain) can be unpredictable via friction energy loss and slipping.

To provide a space of actions that is as directly indicative of robot task needs as possible, but abstract enough to reduce the impact of non-determinism, the concept of "path quality" is introduced. This quality, represented by $X\left(\theta_{i}\right)$, is calculated in varying ways dependent on the algorithm used, but represents the quality of a potential path for the robot to take next. In producing a distribution of quality for all possible paths at each time-step, the state of the environment is represented, and a path can be chosen either via the maximum quality, or by sampling to inject exploration behavior.

\subsection{Rule-Based Navigation}

Rule-based navigation is achieved through a probabilistic path selection algorithm. It is termed "rule-based" because the algorithm does not contain any mechanism for modifying behavior based on knowledge gained during operation. Given a "desired location" (e.g., through a goal selection algorithm or waypoint list) the path selector needs to compute the speed and heading corrections to reach that location.

The definitions for the variables and functions located in the algorithm shown in Figure 2 are as follows:

- $T$ Indexes episodes

- $t$ Indexes time-steps within each episode

- $\theta_{i}$ Angle of potential path $i$

- $\alpha_{\text {des }}$ Vehicle relative angle to destination

- $P_{\text {direct }}$ Probability that the potential path $i$ is direct to the destination

- $P_{\text {safe }}$ Probability that the potential path $i$ is "safe"

- $X\left(\theta_{i}\right)$ Path quality assigned to each potential path

- $\alpha_{u}$ Chosen vehicle relative robot angle for next time-step 


For $T<T_{\text {max }}$ Loop:
For $t<t_{\text {epi }}$ Loop:
Capture current state $s$
For $\theta_{i} \leq 360$ Loop:
1. Calculate $P_{\text {direct }}$ given $\theta_{i}$ and $\alpha_{\text {des }}$
2. Calculate $P_{\text {safe }}$ given $d_{\theta_{i}}$
3. $X\left(\theta_{i}\right) \leftarrow P_{\text {direct }} \times P_{\text {safe }}$
$\alpha_{u} \leftarrow$ argmax $X\left(\theta_{i}\right)$
$V_{u} \leftarrow F\left(X\left(\alpha_{u}\right)\right)$

Figure 2: Rule-Based Navigation Algorithm: Algorithm to determine the quality of potential robot paths. See text body for definitions.

- $F\left(X\left(\alpha_{u}\right)\right)$ Linear mapping of path quality of path chosen to robot speed

- $V_{u}$ Chosen robot speed

In this approach, each path is assigned a probability of leading to the desired location based on the current heading of the robot, provided by inertial sensing. Then, each path is assigned a probability of safety, which is based on the presence of observable obstacles along a given path, provided by ultrasonic sensing. The product of these two probabilities provides a prediction of success for a given path and is labeled the path quality. The path with greatest quality (greatest "probability of success") is then chosen as the, potentially new, desired robot heading.

In the absence of prior information, this is a likelihood based approach, where each path is evaluated solely based on collected data. However, including prior information and updating the path quality posterior probabilities would provide a true Bayesian navigation method. Figure 2 shows the algorithm. The heading with the highest path quality is selected, and the speed is linearly scaled based on that quality (e.g., $90 \%$ or greater path quality equates to maximum speed, whereas a $50 \%$ path quality results in half speed). 


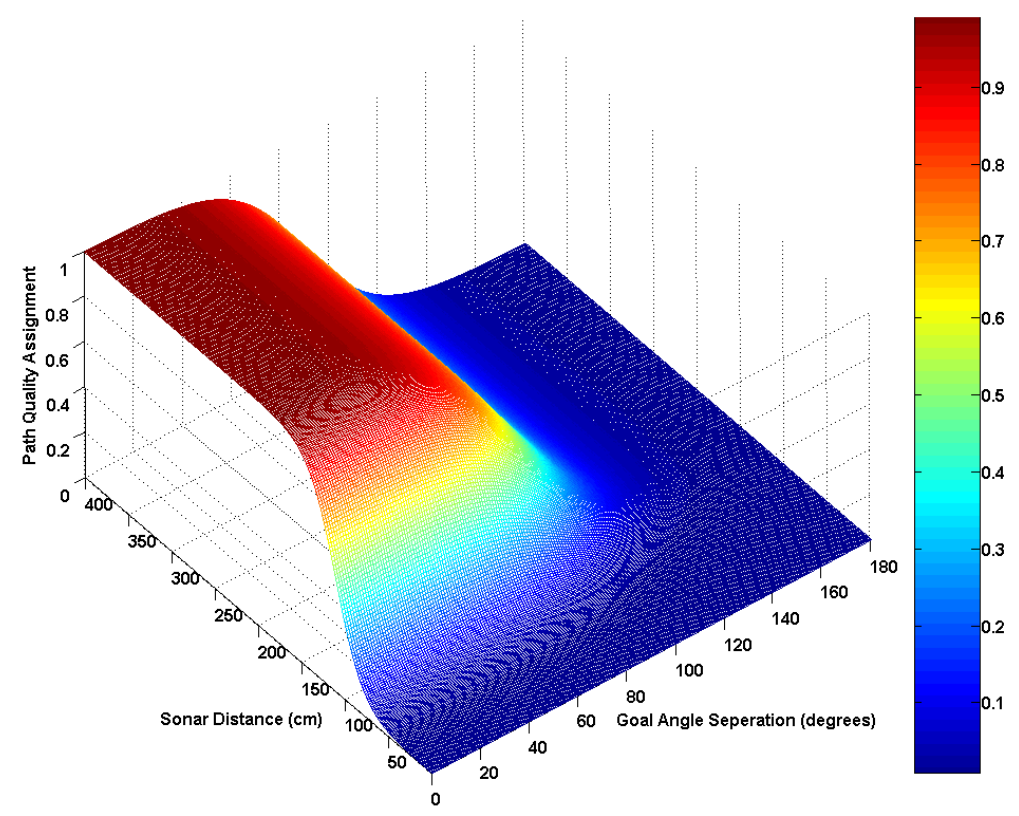

Figure 3: Resulting path quality assignments are shown for the entire range of potential state inputs to the rule-based algorithm. Highest path quality is where there is a large distance to the nearest obstacle on a potential path, and that path points towards the destination (upper left area).

The path quality determination for each potential path is engineered offline based on known robotic articulation capabilities (e.g., speed and turning ability) and on experimental observations made during the initial design. By assigning a quality to each potential path and choosing the best, a smooth transition is made between discrete path choices as the robot navigates through the environment, even for dynamically changing desired locations. For example, the goal selection mechanism (or waypoint list) may deliver a dramatically different desired location at the next time step, however since the navigation algorithm is executed (and a simple path recalculated) at every time step, rather than complex multiple time step paths being designed for new destinations, the navigation commands are minimally affected by the sudden change in destination. 


\subsection{Neuro-Evolution}

The state/action structure of the rule-based navigation control presented in Section 2.2 contains a beneficial approach to path selection. It is simple, which reduces computational complexity as well as the number of potentially unpredictable behaviors, and applies well to a mobile robot with limited sensing capabilities.

Initialize $N$ networks at $T=0$

For $T<T_{\max }$ Loop:

1. Pick a random network $N_{i}$ from population

With probability $\epsilon: N_{\text {current }} \leftarrow N_{i}$

With probability $1-\epsilon: N_{\text {current }} \leftarrow N_{\text {best }}$

2. Mutate $N_{\text {current }}$ to produce $N^{\prime}$

3. Control robot with $N^{\prime}$ for next episode

For $t<t_{\text {epi }}$ Loop:

3.1 For $\theta_{i} \leq 360$ Loop:

Run $N^{\prime}$ to produce $X\left(\theta_{i}\right)$

$3.2 \alpha_{u} \leftarrow \operatorname{argmax} X\left(\theta_{i}\right)$

$3.3 V_{u} \leftarrow F\left(X\left(\alpha_{u}\right)\right)$

4. Rank $N^{\prime}$ based on performance

(objective function)

5. Replace $N_{\text {worst }}$ with $N^{\prime}$

Figure 4: Evolutionary Algorithm: An $\epsilon$-greedy evolutionary algorithm to determine the weights of the neural networks. See text body for definitions.

To capture those benefits while injecting the benefits of adaptability into navigation control, the state and action spaces are maintained. Specifically, one state variable represents the angular distance of a potential path from 
the desired heading, and one represents the distance to the nearest impassable object in that path. The action space then contains a single variable representing the quality of the path given that information. Therefore, for this work, the baseline network structure created is a two layer, sigmoid activated, artificial neural network.

This network is run at each time step, for each potential path, generating a path quality function in a similar fashion to that presented in Figure 2. The difference is in the replacement of static predefined probability distributions by an adaptive artificial neural network.

An evolutionary search algorithm for ranking and subsequently locating successful networks within a population [43, 44, 45] is applied here. The algorithm maintains a population of twenty networks, utilizes mutation to modify individuals, and ranks them based on a performance metric specific to the domain. The search algorithm used is shown in Figure 4 which displays the ranking and mutation steps.

The definitions for the variables and functions located in the algorithm shown in Figure 4 are as follows:

- $T$ Indexes episodes

- $t$ Indexes time-steps within each episode

- $\theta_{i}$ Angle of potential path $i$

- $N$ Indexes networks with appropriate subscripts

- $N^{\prime}$ Mutated network for use in control of current episode

- $X\left(\theta_{i}\right)$ Path quality assigned to each potential path

- $\alpha_{u}$ Chosen vehicle relative robot angle for next time-step

- $F\left(X\left(\alpha_{u}\right)\right)$ Linear mapping of path quality of path chosen to robot speed

- $V_{u}$ Chosen robot speed

In this domain, mutation (Step 2) involves adding a randomly generated number to every weight within the network. This can be done in a large variety of ways, however it is done here by sampling from a random Cauchy distribution [46] where the samples are limited to the continuous range [10.0,10.0]. Ranking of the network performance (Step 4) is done using a domain specific objective function, and is discussed in detail in Section 3. 


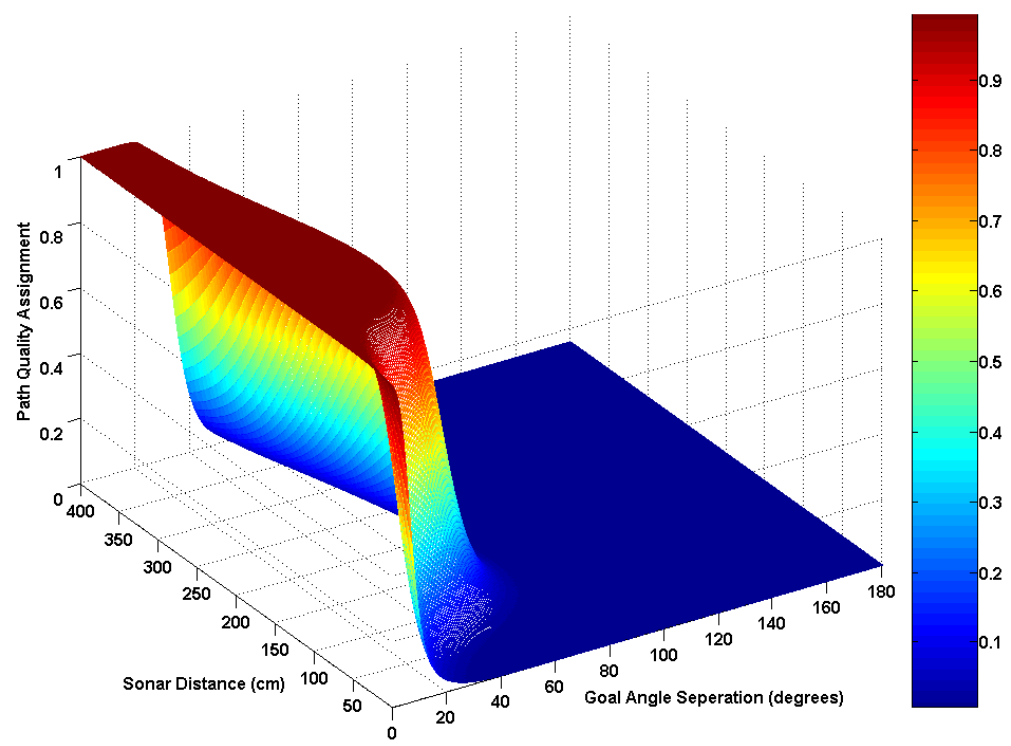

Figure 5: Network outputs for entire range of inputs in the neuro-evolutionary algorithm. The figure is produced after the learning process has converged within a difficult navigation situation (Section 4).

\section{Robot Navigation Problem}

Essential in any robotic exploration domain is the ability to evaluate the performance of techniques used for navigation. In general a performance metric is needed for evaluation and tuning purposes, but specifically when utilizing learning algorithms, an objective function is required for providing the algorithm with a signal indicating success or failure of action decisions made during the learning process. This objective preferably will have a clear gradient such that a learning algorithm can determine in which direction better performance can be achieved. In the work here, a time-extended (system-level) calculation of behavior throughout an entire episode was used to rank networks within the population.

\subsection{Robot Capabilities}

To first conceptually construct the forthcoming objectives, it is important to first examine the robot capabilities in the work for this paper. To meet the goal of adaptive navigation under strong resource limitations, a unique set of 
state and action spaces were developed in Section 2.1. However, those spaces are generally applicable, only providing a general structure for most mobile robots, requiring only that robot motion and general environment detection are available.

For the work presented in this paper, a specific robot platform was chosen on which the physics-based simulations were based. They are broken into sensing and actuation below:

- Sensing: The robot must determine its orientation (position and heading) as well as information regarding the environment surrounding it. This is done in this work via:

- Orientation: The robot contains inertial sensing from which it can integrate its position and heading (from initial orientation). The initial orientation must be known in order to calculate the location of the destination, but knowledge of the environment itself (i.e., obstacles) is not required.

- Environment: Ultrasonic sensing is done to detect the relative locations of obstacles surrounding the robot. Ultrasonics vary widely in field of view and range. For this work, 8 sensors are used to provide a 360 degree field of view in 45 degree increments. The range of the sonar units is 4 meters.

- Actuation: The robot has two motorized wheels and a caster. Therefore, it is capable of moving in any direction, either by turning in place via differential wheel motion, or slowing a wheel to turn while in motion. The maximum accelerations and speeds are based on a physical robot model.

As a result of this robot model, the amount of information is strongly limited in state, particularly the resolution in which the robot can observe the surrounding environment. In addition, while actuation does provide significant articulation capabilities on flat surfaces, there are several levels of control hidden from the navigation algorithms, and therefore the result of action choices may not be deterministic.

With these robot capabilities in mind, we can now examine the objective design, both at the system level and at the robot level to further understand the detail regarding desired robot behavior and the impact of state changes (or lack thereof) on action results. 


\subsection{Learning Objective}

An episodic objective aims to capture three important aspects of mobile robot navigation in unknown environments under the capability restrictions described above; 1) total path length the robot uses to reach the destination, 2) time the robot consumes reaching the destination, and 3) time the robot consumes recovering from a collision with an obstacle. These incorporate choosing the shortest path, executing it with greatest speed, and doing so in a safe manner. In order to convert the above to maximization rather than minimization, and support constantly shifting initial conditions, the best possible behavior is incorporated, generating the following objective function:

$$
R(s)=\alpha\left(d_{\text {best }}-d_{\text {actual }}\right)+\beta\left(t_{\text {best }}-t_{\text {actual }}\right)-\gamma t_{\text {collision }}
$$

where $d$ is the path length (best possible and episode actual), $t$ is the time consumed, and $\tau_{\text {collision }}$ is the total amount of time spent recovering from collisions. The best possible of these is used to indicate what would happen if the robot took a straight path, at maximum velocity, without hitting any obstacles. $\alpha, \beta$ and $\gamma$ are constants used to increase or decrease the respective terms' contribution to the overall function.

\section{Simulation Experiments}

Several experiments were designed to evaluate the navigation algorithms for a specific set of behaviors discussed in the problem definition. These progressively increased in difficulty and scope from basic navigation to a destination, through advanced navigation in cluttered environments. In all experiments, an arena of 5 meters square was created with a varying number of obstacles, depending on the experiment. The learning method is episodic in that the robot is allowed to operate for a fixed maximum amount of time $\left(t_{e p i}=60\right.$ seconds in this work). Learning is executed for 2000 episodes, and each experiment is run 40 times for each algorithm. Following is a list of navigation experiments with their intentions:

- No Obstacles: The robot must simply navigate to a destination with no obstacles between the robot and the destination. This experiment evaluates the ability for the algorithms' to effectively control robot articulation. 
- Fence Obstacles: The robot must navigate first through an open environment, then is suddenly presented with a "fence" of obstacles. This experiment evaluates the algorithms' ability to handle emergent difficult situations during navigation.

- Dense Environment: The robot must navigate entirely through a dense environment, scattered randomly with obstacles. This experiment evaluates the algorithms' ability to navigate a complex environment rich with sensor information.

- Dense Environment w/ Noise: The robot must navigate entirely through a dense environment while experiencing random sensor and actuator noise. This experiment evaluates the algorithms' robustness to real-world situational signal noise.

These experiments evaluate not only the navigation algorithm's ability to seek a destination, but safely and intricately navigate around obstacles in an unknown environment, including when state information is inaccurate and action results are stochastic.

\subsection{No Obstacles}

In these experiments the algorithms are evaluated for simple and intricate navigation techniques. The first of these determines ability to relocate to a destination and the initial conditions are shown in Figure 6. The structure of this figure is utilized throughout the results presentation. It shows the to-scale experiment arena with sample robot (square) and destination (diamond). The light grey area (yellow in a color printing) shows the possible starting locations for each episode, the number of which is listed at the base of the arena.

Figure 6 shows the learning results for the No Obstacles situation. The objective function shown in Equation 1 is plotted over learning episodes as an average over the 40 iterations for each algorithm. The random driver, rule-based driver, and neuro-evolution algorithms are provided with error bars indicating the standard deviation of the data. As shown, the random driver sets a good baseline by taking random actions. The rule-based driver does very well as all starting positions produce direct paths without the need for obstacle avoidance.

The neuro-evolutionary algorithm is shown to learn quickly, performing statistically similar to the rule-based driver within approximately 600 

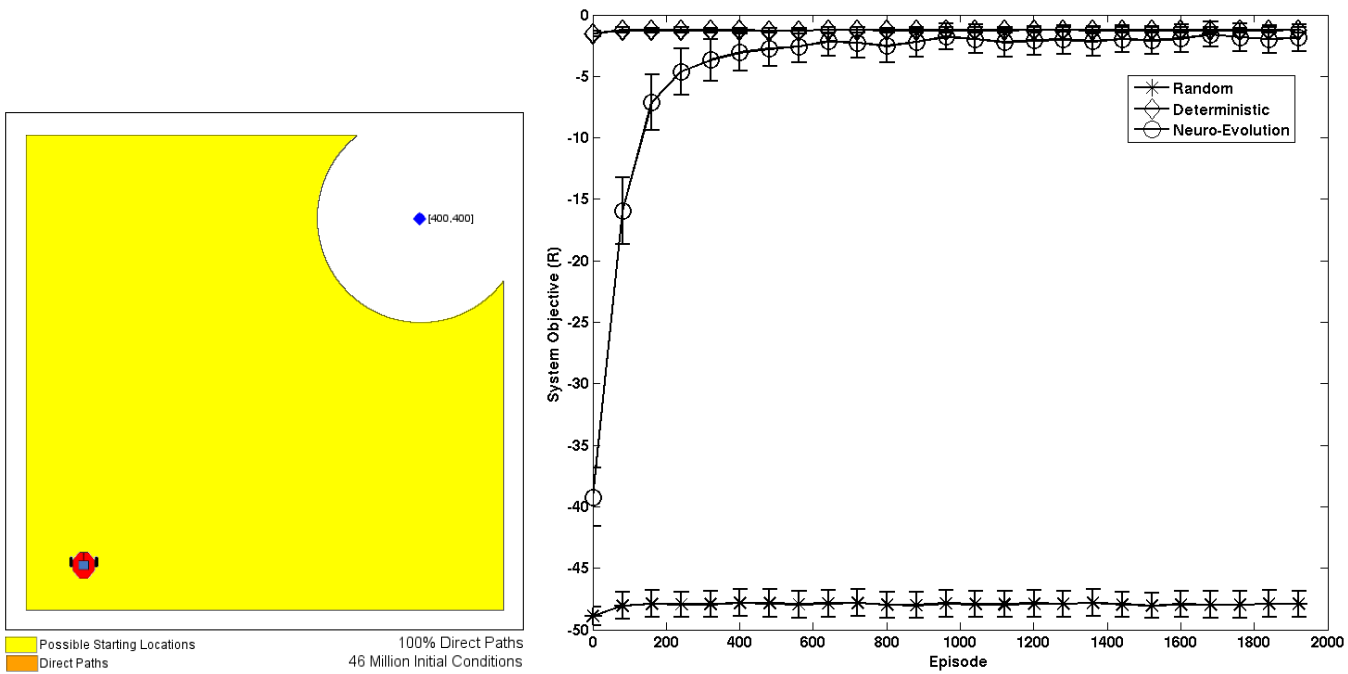

Figure 6: Left: The experimental arena for the No Obstacles situation. The figure is to scale and shows a sample robot (square), the destination (diamond) as well as the possible starting locations for an episode (light grey/yellow area). The number of possible initial conditions as well as the approximate percentage of those being direct paths are listed at the base of the figure. Right: The results of the learning for the No Obstacles situation. The objective function is plotted for the random, rule-based, and neuro-evolutionary algorithms as an average over 40 iterations.

episodes. Because the adaptive navigation technique inherently contains some exploration, the mean is below rule-based navigation in very simple situations. For example, the rule-based navigator drives directly to the destination, where the neuro-evolutionary technique chooses random policies with $\epsilon$ probability which, even after convergence, may be slightly less performing policies.

\subsection{Fence Obstacles}

A difficult situation was configured next where there were few potential direct paths to the destination and the robot is required to navigate carefully between obstacles in order to reach the destination. Figure 7 shows the layout of the obstacles as they relate to potential robot starting positions.

The results, shown in Figure 7, reflect the difficulty of the situation. Observable in Figure 7 (left) is the very small separation between obstacles. In fact, the distance is not significantly larger than the width of the robot, 

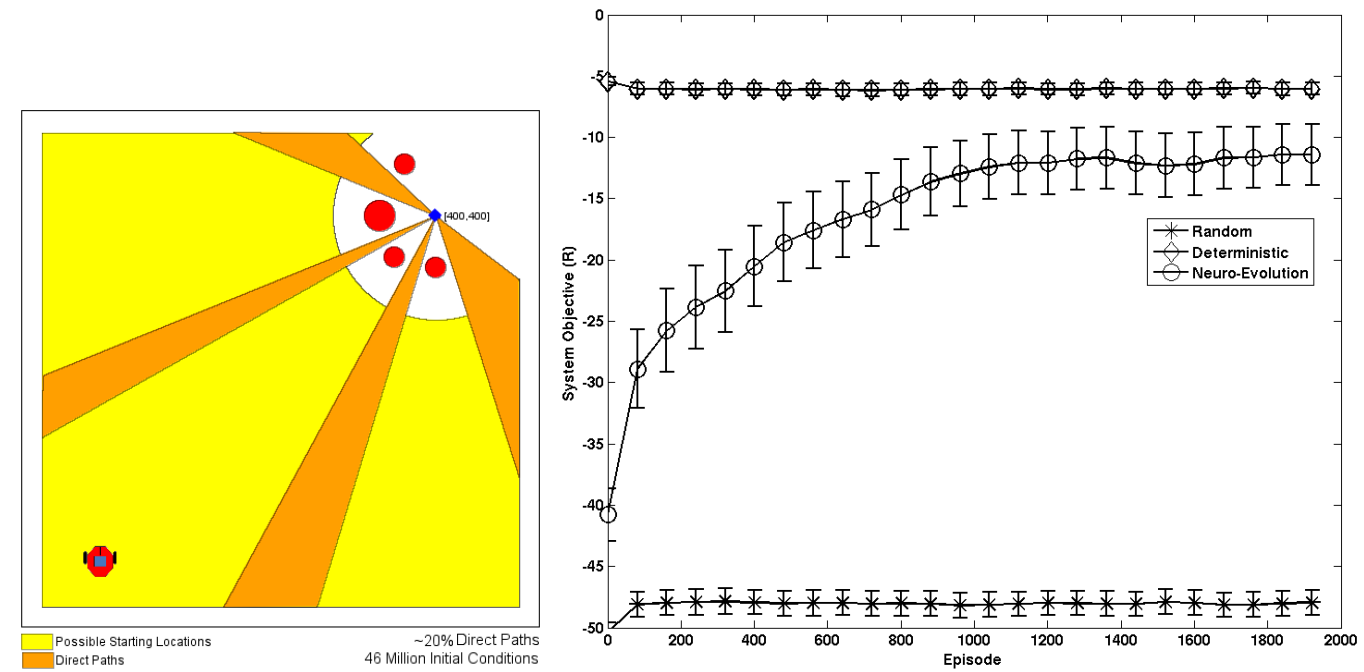

Figure 7: Left: The experimental arena for the Fence Obstacles situation. The figure is to scale and shows a sample robot (square), the destination (diamond) as well as the possible starting locations for an episode (light grey/yellow area). The number of possible initial conditions as well as the approximate percentage of those being direct paths are listed at the base of the figure. Right: The results of the learning for the Fence Obstacles situation. The objective function is plotted for the random, rule-based, and neuro-evolutionary algorithms as an average over 40 iterations.

requiring the algorithms to learn to navigate very carefully to not collide with an obstacle. The rule-based driver performs consistent to the previous situation, however the adaptive algorithm appears to have trouble discovering optimal solutions to such a difficult situation.

The neuro-evolution algorithm learning curve is damped, as this situation presents three stages of learning. The algorithm must first learn to navigate toward the destination, is then presented with densely placed obstacles to avoid, and finally must learn to do both at decent speed. The neuro-evolution algorithm converges to a solution $50 \%$ lower than that of the rule-based navigator.

\subsection{Dense Environment}

The next step in the progression of analysis was to determine how the algorithms perform in a complex environment dense with obstacles. It is desirable to force the robot to navigate through the majority of the environment and reduce or eliminate the number of potential direct paths. To 

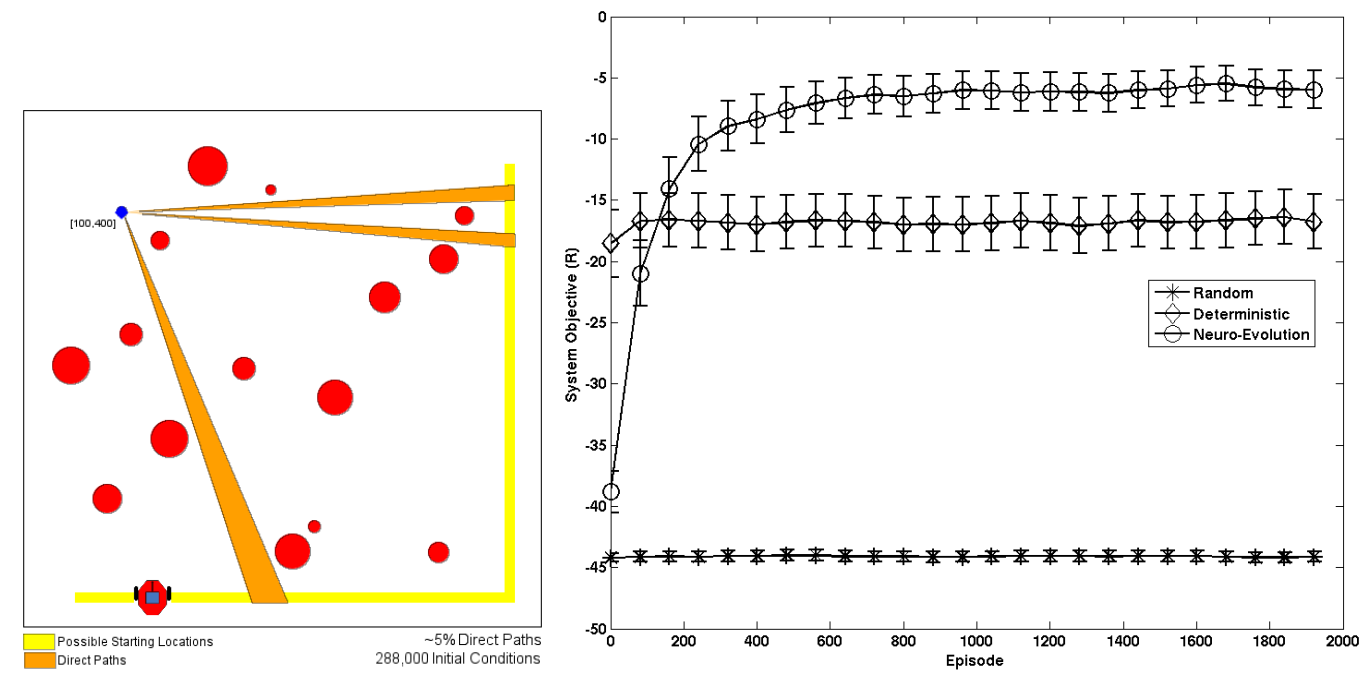

Figure 8: Left: The experimental arena for the dense environment. The figure is to scale and shows a sample robot (square), the destination (diamond) as well as the possible starting locations for an episode (light grey/yellow area). The number of possible initial conditions as well as the approximate percentage of those being direct paths are listed at the base of the figure. Right: The result of the learning in a dense environment. The objective function is plotted for the random, rule-based, and neuro-evolutionary algorithms as an average over 40 iterations.

provide this, the range of starting locations was limited to areas on the opposing side of the experimental arena and 15 obstacles were placed arbitrarily throughout the environment. Figure 8 shows the environment configuration for these experiments. As shown, the starting positions are narrowed to the outer edges of the arena, but the robot initial heading was still randomized at the beginning of each episode.

The results in this environment are very interesting. The neuro-evolutionary algorithm learns more quickly than in the situational experiments and exceeds the reduced performance of rule-based navigation. This is a result of the algorithm being presented with more information during the learning process (through richer sonar data), the reduced number of starting positions, and the discovery of more accurate obstacle avoidance technique over rule-based navigation. The results of the learning process are presented in Figure 8 . 


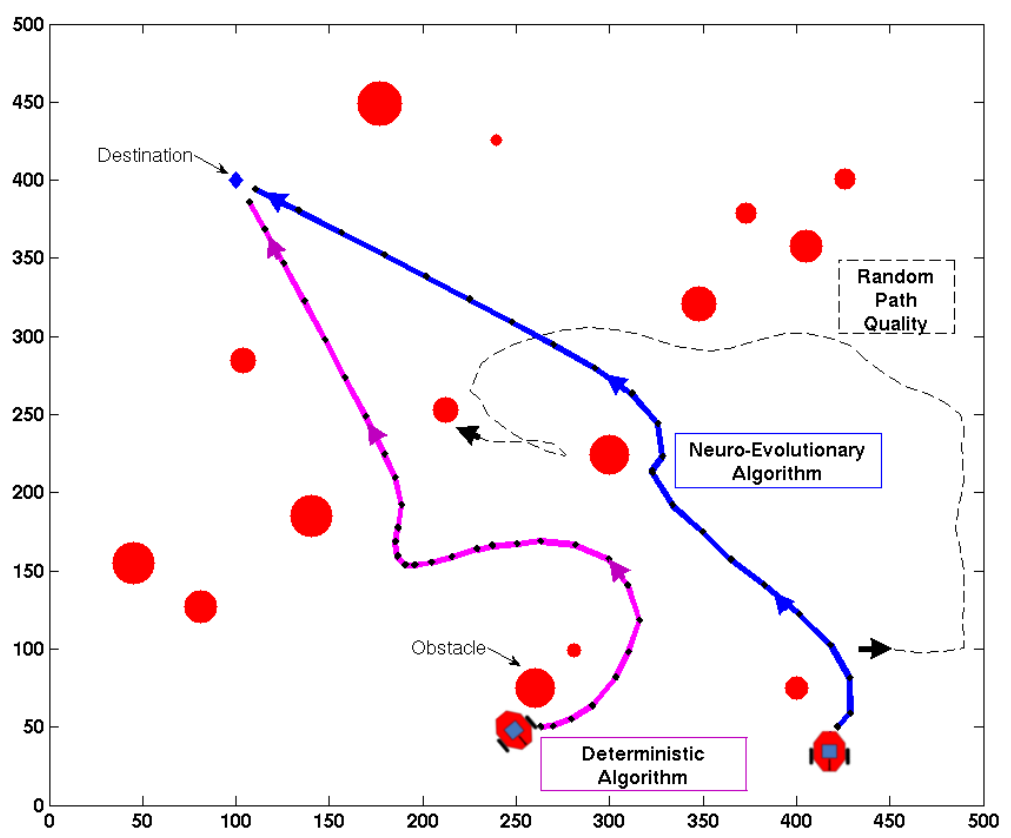

Figure 9: Sample robot paths are displayed. Figure is generated using data produced during runs with converged networks. Initial placement and heading is randomized at every iteration, so paths were selected that began in approximately the same location. Circles are obstacles, and robot orientation on each path indicate the robot initial heading.

\subsection{Dense Environment Paths}

Throughout the experimental process, the behavior of the mobile robots for each algorithm were carefully examined to ensure not only that appropriate behavior was being learned, but also to determine areas for potential algorithmic improvements. Shown in Figure 9 are sample paths through the dense environment presented in the previous section. The paths are sampled after learning convergence is achieved and plotted in 1 second real-time intervals (black stars on paths).

Unique behavior characteristics are present in all navigation techniques. First, in the rule-based path it is clear that obstacle avoidance is favored, where the midpoint between two obstacles is chosen as often as possible when turning toward the destination. The neuro-evolutionary algorithm on the other hand learns to favor directness slightly more than safety, traversing as close to obstacles as is safe in order to minimize ground covered. 


\subsection{Impact of Obstacle Density}

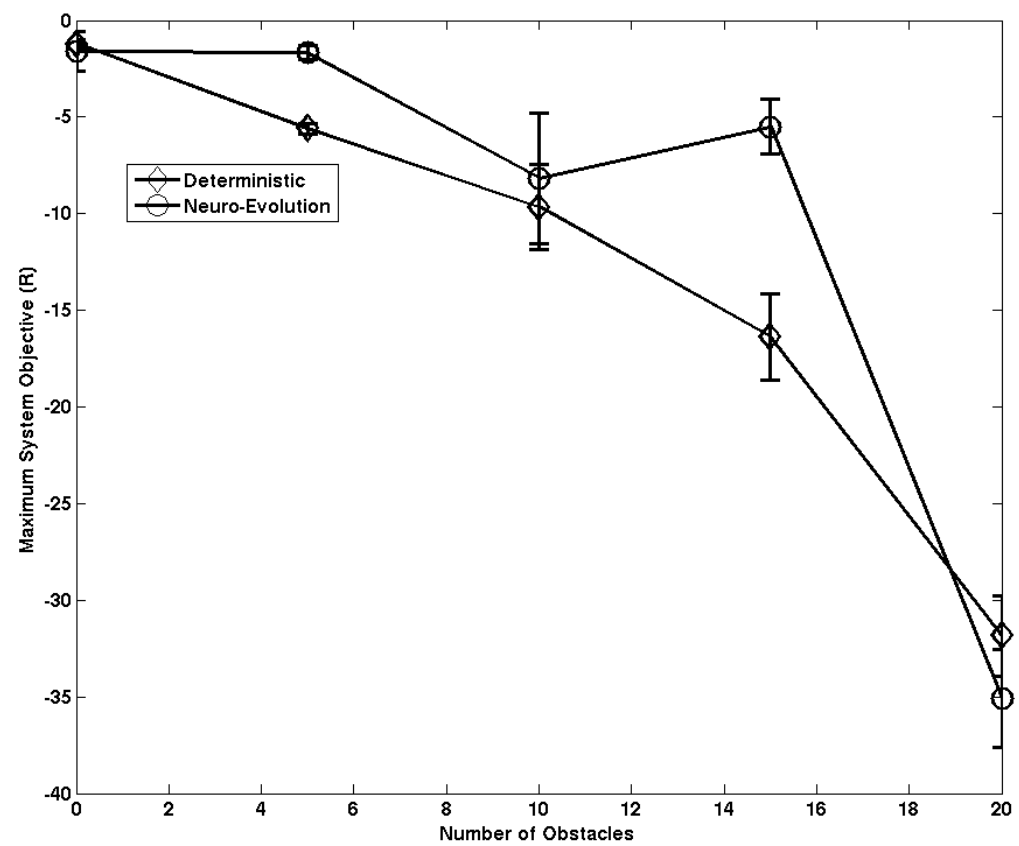

Figure 10: The impact of obstacle density is shown. Maximum system objective achieved is plotted against varying number of obstacles within the environment.

The idiosyncrasies in learned behavior above point to possible hidden aspects involved in the interpretation of the information received via sensors. Of particular interest is the reaction of algorithms to the density of obstacles within the environment. With limited environment detection capabilities, as the environment becomes more dense with hazards, the robot must be careful with path quality assignments such that safe operation is ensured.

Figure 10 shows the impact of obstacle density on the performance of the algorithms of interest. As expected, all algorithms lose performance with increasing number of obstacles, however it is particularly interesting to note the degree in which they lose performance. The rule-based algorithm consistently looses performance as the environment increases in density with a fairly sharp slope. The neuro-evolutionary algorithm is able to relatively maintain performance, but sharply declines between 15 and 20 obstacles, even unable to locate the destination on occasion within the time allotted. 


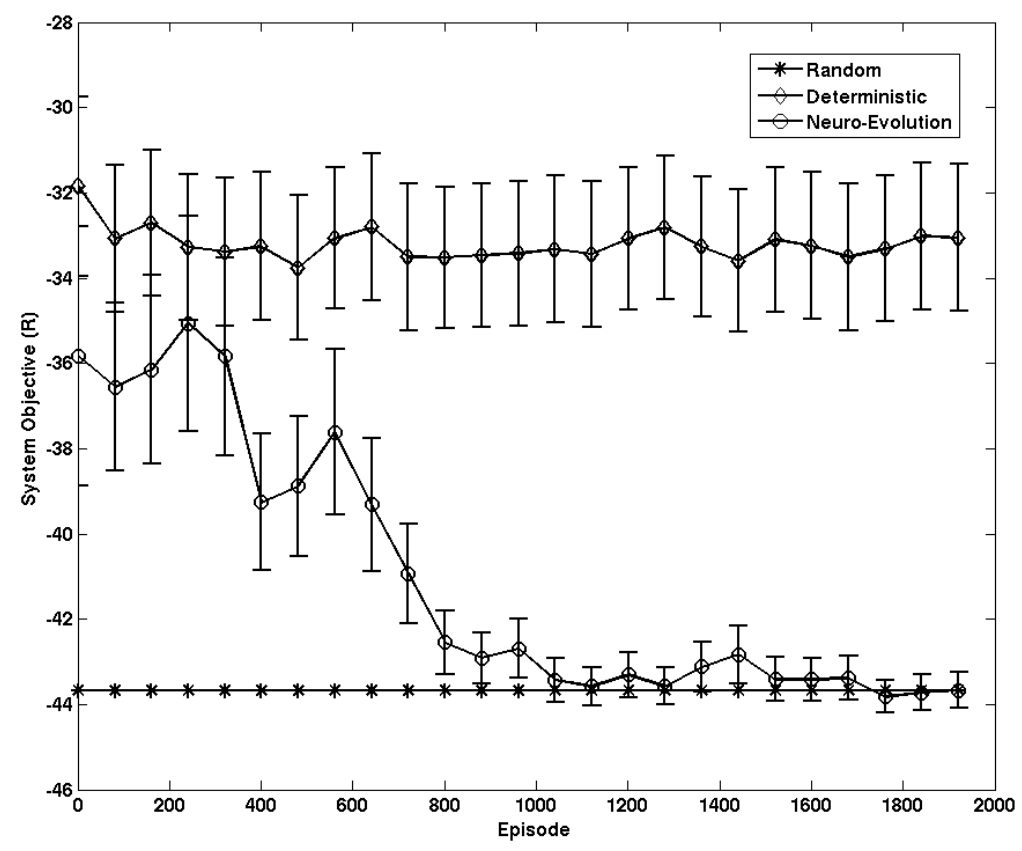

Figure 11: The result of the learning in a dense environment containing 20 obstacles. The objective function is plotted for the random, rule-based, and neuro-evolutionary algorithms as an average over 40 iterations.

The performances are of course strongly affected by the resolution of environment sensing capabilities. The environment containing 20 obstacles was too cluttered to be accurately represented by the sensing capabilities, and therefore all navigation algorithms broke down. A detailed examination of the impact of sensing capability is done in other work.

\subsection{Dense Environment with Noise}

Previously the sensors and actuators produced ideal data and robot motion. This is not a realistic situation for physical robots, as all sensors contain stochastic differences in readings of the environment, and actuators may not produce exactly the intended robot motion. Therefore, random noise was injected into the sonar and inertial sensor data as well as the output of the navigation algorithm to the actuators. Specifically, 5\% random noise was present from the beginning of the learning and to simulate potential failures, the noise level was phased up to $10 \%$ over 200 episodes surrounding 
the 1000th episode (e.g., from 900 to 1100). The results of the learning are presented in Figure 12.

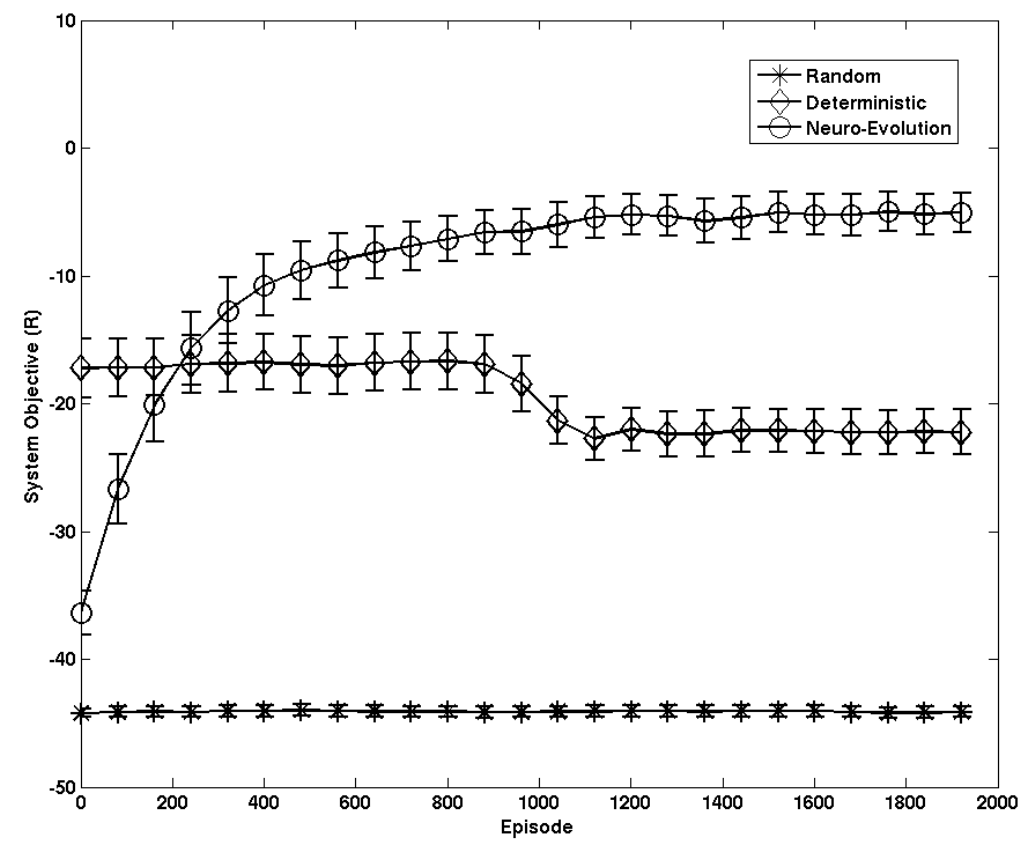

Figure 12: The results of the learning in a dense environment with 15 obstacles while sensor and actuator noise was present. The objective function is plotted for the random, rulebased, neuro-evolutionary, and policy gradient algorithms as an average over 40 iterations.

It can be seen that the learning process is dampened as a result of the noise present at the beginning of the learning process. It is important to note that as the increased noise is phased in, there is little or no effect in the performance of the neuro-evolutionary algorithm. Learning continues unimpeded to significantly outperform the rule-based navigation algorithm which is strongly affected by the increased noise level in sensing and actuation. This is a result of the learning occurring while noise is present in the system such that good behavior is learned in spite of the noise and therefore an increase in the noise level during operation (once successful behavior has been learned) does not affect the neuro-evolutionary algorithm performance.

\subsection{Impact of Signal Noise}

The apparent ability of the adaptive algorithm to learn successful behavior in the face of signal noise is an interesting result. To examine this 


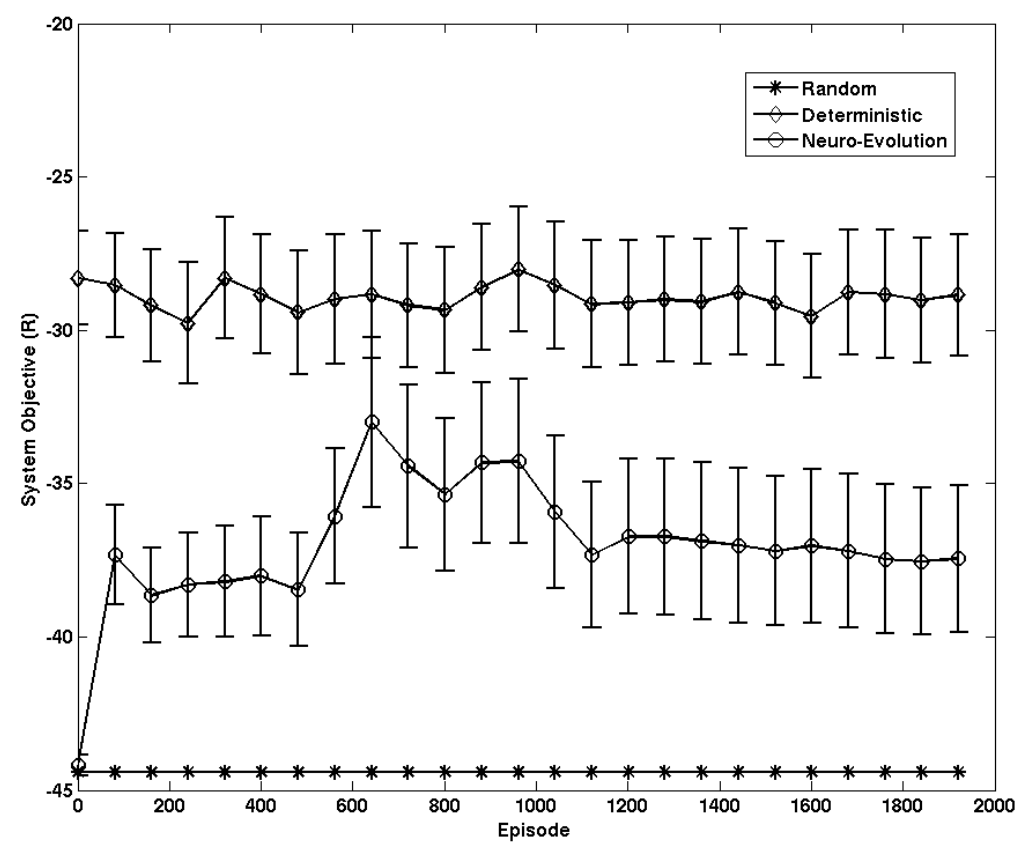

Figure 13: The results of the learning in a dense environment with 20 obstacles while sensor and actuator noise was present. The objective function is plotted for the random, rule-based, and neuro-evolutionary algorithms as an average over 40 iterations.

ability further, an experiment was done to evaluate the degree to which the algorithms are able to operate when the robot begins to fail.

Shown in Figure 14 are the results of increasing signal noise on the algorithms' performance. Here the neuro-evolution algorithm is the best performer overall, able to maintain good performance until the noise level reaches $20 \%$, at which point performance falls away significantly.

This behavior is a direct result of the objective used for learning. The neuro-evolution algorithm utilizes a general system-level performance metric, and therefore is able to learn good behavior regardless of the complications within robot sensing and articulation, likely encoding the signal noise as a part of the knowledge gained during learning.

\section{Hardware Experiments}

The simulator for the experiments in the above sections was a physicsbased simulator designed to run in an episodic nature, such that learning 


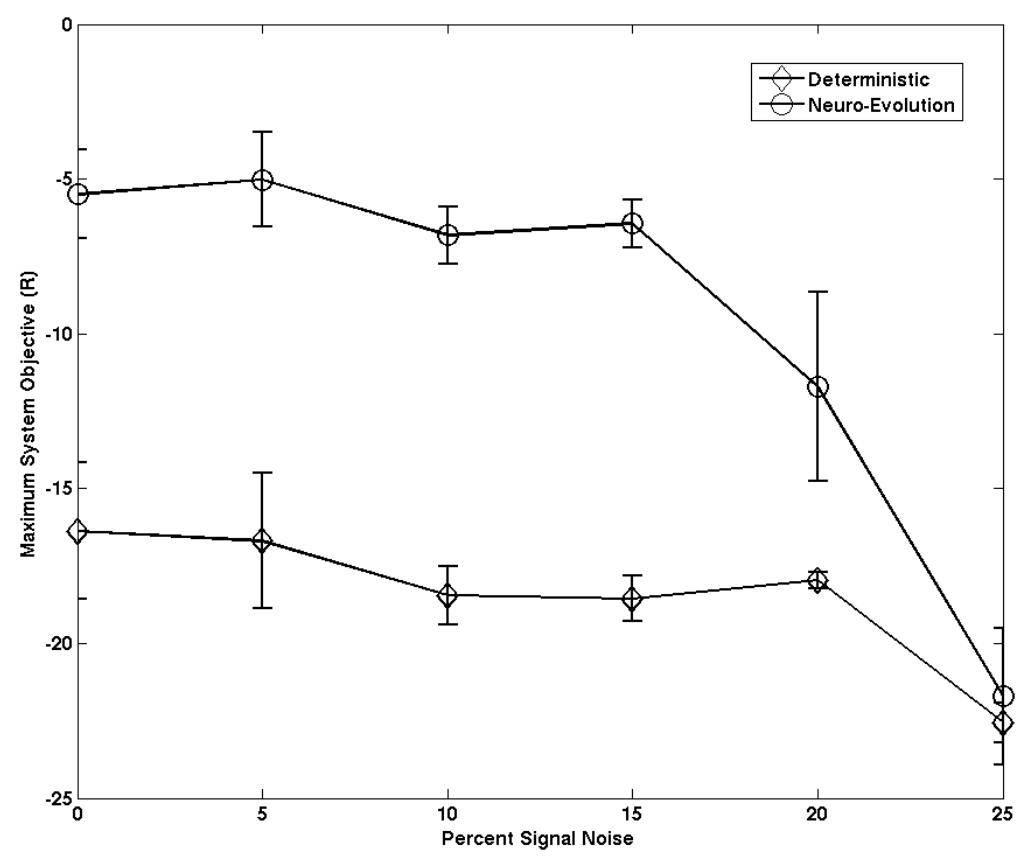

Figure 14: The impact of signal noise is shown. Maximum system objective achieved is plotted against varying noise levels.

algorithms could be trained and evaluated quickly. The robot platform on which that simulator was based is different from the one discussed in this section, primarily due to the origins (the robot platform discussed here was decided on later in the research). However, it still utilized sonar units for environment detection and was a differential steering robot platform. The primary differences are in the size of the platform, and the acceleration and deceleration capabilities.

With the robot platform discussed here, a simulator called MobileSim is shipped by the manufacturer. It is based on the popular Player/Stage robot simulation platform, however has been heavily modified to represent the dynamics of the Pioneer $3 D X$ robot platform. The MobileSim software emulates the operating system and is an extremely accurate representation of robot behavior. So much so that interface software can connect directly to it and "speak" to the software as if it were the real robot, and therefore does not know whether it is currently operating on a real platform or within a simulated environment. As a result of this however, the simulator must run 


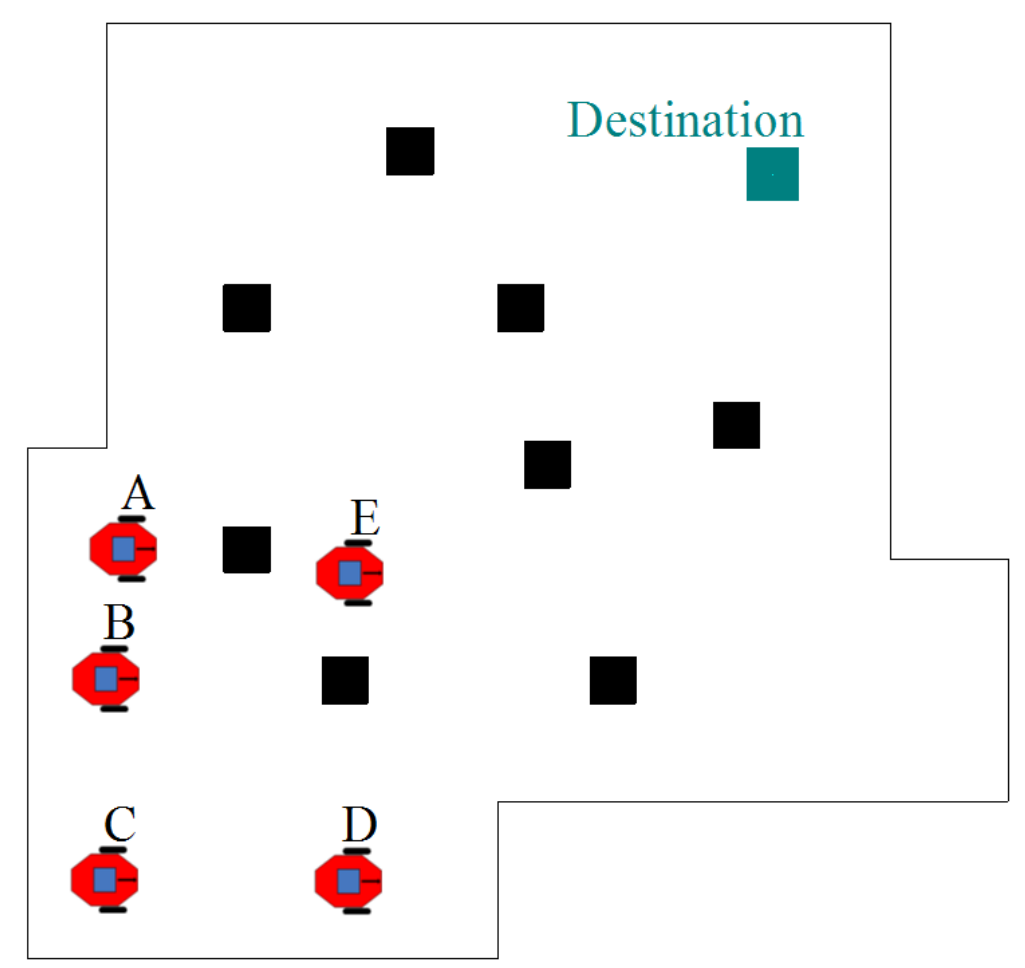

Figure 15: Figure showing the environment used for functional experiments. The figure is to-scale and represents the Autonomous Systems research lab. Obstacles are solid black squares and are 14" on each side. Also shown are the 5 sampled starting locations and the destination.

in real-time, resulting in the training of episodic learning algorithms being intractable.

An experiment area was designed for testing the navigation algorithms discussed in previous sections. The area is shown in Figure 15 which represents the Autonomous Systems laboratory. It contains several static obstacles that the robot is required to navigate through to reach a destination. Several starting locations were created to ensure that various paths were required to complete the experiment. The robot heading was randomized for each iteration (a total of 10 for each starting location).

\subsection{Rule-Based Navigation}

The rule-based navigation algorithm, discussed in Section 2.2, maintained the same structure and capabilities after installation on the new platform. 
However, with significant changes in sensing and articulation capabilities, the algorithm parameters required significant tuning to establish the same level of performance as demonstrated in the original simulator. This was to be expected, as the parameters were originally tuned based on a different platform and with different sensing resources. The equivalent level of performance was established however, as can be seen in the results shown in Table 1. The functional experiment results show, while the numbers differ slightly due to the change in environment and platform, similar performance is achieved after tuning the deterministic algorithm parameters. These parameters primarily include the probabilistic distributions, but the speed control function needed modification as well.

\subsection{Neuro-Evolutionary Navigation}

In sharp contrast to the rule-based algorithm, the adaptive navigation algorithm used in the above sections of this paper required no tuning themselves. The tuned speed controller was left in place from the rule-based algorithm experiments, to maintain comparability, but no other algorithm parameters were changed. Indeed, the neural network that was trained in the original simulation platform was used directly, and the algorithm simply had learning removed (feed-forward network usage only). The results are shown with the rule-based algorithm in Table 1.

\begin{tabular}{|l|r|r|r|r|r|}
\hline Algorithm & A & B & C & D & E \\
\hline \hline Rule-Based (sim) & $-8.8 \pm 0.3$ & $-11.4 \pm 1.0$ & $-9.5 \pm 0.4$ & $-8.1 \pm 0.1$ & $-6.5 \pm 0.2$ \\
\hline Rule-Based (real) & $-8.2 \pm 0.4$ & $-8.1 \pm 0.3$ & $-8.3 \pm 0.3$ & $-8.6 \pm 0.3$ & $-7.1 \pm 0.4$ \\
\hline \hline Neuro-Evolution (sim) & $-12.9 \pm 2.7$ & $-9.1 \pm 2.5$ & $-6.4 \pm 0.4$ & $-6.2 \pm 0.2$ & $-3.5 \pm 0.2$ \\
\hline Neuro-Evolution (real) & $-5.6 \pm 0.5$ & $-6.9 \pm 1.1$ & $-5.4 \pm 0.2$ & $-6.2 \pm 0.3$ & $-5.9 \pm 0.8$ \\
\hline \hline
\end{tabular}

Table 1: The results of experimentation for both navigation algorithms. The numbers shown are the same system evaluation $R(s)$ discussed in Section 3.2 and shown in Section 4.

While the performances are all similar to those in the previous simulations, and are similar among algorithms here, the most striking result is the capability of the algorithm across platforms. The adaptive algorithm was trained on one (while similar) robot platform and implemented on another without modification. This points directly to the features of the unique state and action space developed. Rather than requiring of the learning algorithm to map directly between states and actions, the algorithm was required to determine the quality of the surrounding environment for travel, and therefore it did not matter how the path for travel was executed. This provides a 
strong and platform independent methodology for adaptive navigation with autonomous robots.

It is also important to note that the neuro-evolutionary algorithm incurred the fewest time in handling collisions. The robot is prevented from actually colliding with anything at the interface level, to avoid physical damage, but the interface tracks how long that it spent recovering from such an event and reports it. Therefore, if an algorithm of any kind comes too close to an obstacle in the direction of robot travel, the interface interrupts the control process and rotates the robot to a more appropriate orientation and releases control back to the navigation layer. While all algorithms had similar time spent in recovering from collisions (less than 10 seconds), the neuro-evolution algorithm spent $9 \%$ on average less time recovering than the rule-based algorithm.

\section{Discussion}

Robotic exploration is an attractive area of research for several reasons, certainly not limited to cost effectiveness and safety. If a robot is provided with proper capabilities, the amount of information that can be gathered and time in which the gathering takes place can both be improved significantly. Planetary exploration and search and rescue robotics are of great interest to the work presented in this paper, particularly in situations where a robot must navigate through an unknown and partially observable environment.

The robotic platform used in this work was not allowed to maintain a detailed map of its environment, and therefore was required to make decisions based on information immediately available using a limited encoding of experience via simple artificial neural networks. To provide adaptive and robust navigation under such conditions, we used a unique state/action mapping. Neuro-evolutionary navigation provided better overall behavior than rule-based navigation where the system designer provided a set of strategies for actions.

The work is this paper demonstrates that in limiting a robot to only the amount of resources exactly required of it to complete the navigation task, adaptive behavior can still be successful, indeed can perform better in the face of sensor and actuator failures than techniques based on set probability distributions. The neuro-evolutionary algorithm learned slowly, but converged to higher performance as it was capable of learning on an objective more directly indicative of system-level requirements. 
Conversely, when the environments became particularly complex, the neuro-evolutionary algorithm was not able to establish a good policy for navigation. This was due to the difficulty of the time-extended objective function to provide sufficient information based on limited sensing to select

good actions. As the density of the environment increased, specific sets of actions were required at every time-step, and limited sensing prevented the algorithm from identifying where, or even if, those actions were needed.

\section{Acknowledgements}

This work was partially supported by AFOSR grant FA9550-08-1-0187 and NSF grant IIS-0910358.

\section{References}

[1] D. M. Helmick, S. I. Roumeliotis, Slip-compensated path following for planetary exploration rovers, Advanced Robotics 20 (2006) 1257-1280.

[2] C. Kunz, C. Murphy, Deep sea underwater robotic exploration in the ice-covered arctic ocean with auvs, in: Intelligent Robots and Systems, IEEE/RSJ International Conference on, 2008.

[3] J. Bohren, T. Foote, Little ben: The ben franklin racing teams entry in the 2007 darpa urban challenge, Field Robotics Research 25 (2008) $588-614$.

[4] P. Fabiani, V. Fuertes, Autonomous flight and navigation of vtol uavs: from autonomy demonstrations to out-of-sight flights, Aerospace Science and Technology 11 (2007) 183-193.

[5] S. Thrun, G. Sukhatme, Robotics: Science and Systems I, MIT Press, 2005.

[6] S. Thrun, M. Montemerlo, Stanley: The robot that won the darpa grand challenge, Robotic Systems 23 (2006) 661-692.

[7] M. Cummins, P. Newman, Probabilistic appearance based navigation and loop closing, in: Robotics and Automation, IEEE International Conference on, 2007. 
[8] J. A. Fernandez-Leon, G. G. Acosta, M. A. Mayosky, Behavioral control through evolutionary neurocontrollers for autonomous mobile robot navigation, Robotics and Autonomous Systems 57 (2008) 411-419.

[9] F. Gomez, R. Miikkulainen, Solving non-markovian control tasks with neuroevolution, in: Proceedings of the International Joint Conference on Artificial Intelligence (IJCAI-99), Stockholm, Sweden, 1999, pp. 13561361.

[10] A. K. Agogino, K. Tumer, Efficient evaluation functions for evolving coordination, Evolutionary Computation 16 (2) (2008) 257-288.

[11] D. W. van Krevelen, G. S. Nitschke, Neuro-evolution for a gathering and collective construction task, in: Proceedings of the Conference on Genetic and Evolutionary computation, 2008.

[12] A. Agogino, K. Tumer, Evolving distributed agents for managing air traffic, in: Proceedings of the Genetic and Evolutionary Computation Conference, London, UK, 2007.

[13] K. O. Stanley, R. Miikkulainen, Evolving neural networks through augmenting topologies, Evolutionary Computation 10 (2002) 99-127.

[14] T. Balch, R. C. Arkin, Communication in reactive multiagent robotic systems, Autonomous Robots 1 (1) (1994) 27-52.

[15] S. Koenig, C. Tovey, Y. Smirnov, Performance bounds for planning in unknown terrain, Artificial Intelligence 147 (1-2) (2003) 253-279.

[16] K. Rajan, D. Bernard, G. Dorais, E. Gamble, B. Kanefsky, J. Kurien, W. Millar, N. Muscettola, P. Nayak, N. Rouquette, Remote agent: An autonomous control system for the new millennium, ECAI 14 (2000) 726-730.

[17] S. Chien, R. Sherwood, D. Tran, B. Cichy, G. Rabideau, R. Castano, A. Davis, Using autonomy flight software to improve science return on earth observing one, Aerospace Computing, Information, and Communication 2 (2005) 196-216.

[18] A. Mudgal, C. Tovey, S. Koenig, Analysis of greedy robot-navigation methods, in: Proceedings of the International Symposium on Artificial Intelligence and Mathematics (AMAI), 2004. 
[19] A. Mudgal, C. Tovey, S. Greenberg, S. Koenig, Bounds on the travel cost of a mars rover prototype search heuristic, SIAM Journal on Discrete Mathematics 19 (2) (2005) 431-437.

[20] S. Koenig, M. Likhachev, Fast replanning for navigation in unknown terrain, Transactions on Robotics 21 (3) (2005) 354-363.

[21] D. Goldberg, M. J. Mataric, Maximizing reward in a non-stationary mobile robot environment, Autonomous Agents and Multi-Agent Systems 3 (6) (2003) 281-316.

[22] S. Whiteson, P. Stone, K. O. Stanley, R. Miikkulainen, N. Kohl, Automatic feature selection via neuroevolution, in: Proceedings of the Genetic and Evolutionary Computation Conference, 2005.

[23] B. Clement, E. Durfee, Theory for coordinating concurrent hierarchical planning agents, in: Proceedings of the National Conference on Artificial Intelligence, 1999, pp. 495-502.

[24] D. Fox, W. Burgard, S. Thrun, Markov localization for mobile robots in dynamic environments, Journal of Artificial Intelligence Research 11 (1999) 391-427.

[25] S. Thrun, D. Fox, W. Burgard, Monte carlo localization with mixture proposal distribution, in: AAAI National Conference on Artificial Intelligence, AAAI Press / The MIT Press, 2000, pp. 859-865.

[26] S. Thrun, W. Burgard, D. Fox, Probabilistic Robotics, The MIT Press, Cambridge, MA, 2005.

[27] J. Bagnell, S. Kakade, A. Ng, J. Schneider, Policy search by dynamic programming, in: Neural Information Processing Systems, Vol. 16, MIT Press, 2003.

[28] D. Floreano, F. Mondada, Automatic creation of an autonomous agent: Genetic evolution of a neural-network driven robot, in: Proc. of Conf. on Simulation of Adaptive Behavior, 1994.

[29] F. Gomez, R. Miikkulainen, Active guidance for a finless rocket through neuroevolution, in: Proceedings of the Genetic and Evolutionary Computation Conference, Chicago, Illinois, 2003. 
[30] A. Y. Ng, A. Coates, M. Diel, V. Ganapathi, J. Schulte, B. Tse, E. Berger, E. Liang, Inverted autonomous helicopter flight via reinforcement learning, in: International Symposium on Experimental Robotics, 2004.

[31] C. McGann, F. Py, K. Rajan, J. Ryan, R. Henthorn, Adaptive control for autonomous underwater vehicles, in: Assoc. for the Advancement of Artificial Intelligence (AAAI), 2008.

[32] R. S. Sutton, A. G. Barto, Reinforcement Learning: An Introduction, MIT Press, Cambridge, MA, 1998.

[33] M. S. Erden, K. Leblebiciolua, Free gait generation with reinforcement learningnext term for a six-legged robot, Robotics and Autonomous Systems 56 (2008) 199-212.

[34] M. E. Aydina, E. ztemel, Dynamic job-shop scheduling using reinforcement learningnext term agents, Robotics and Autonomous Systems 33 (2000) 169-178.

[35] Lucas, Axel, Active object recognition by view integration and reinforcement learning, Robotics and Autonomous Systems 31 (2000) 71-86.

[36] P. Bartlett, J. Baxter, Stochastic optimization of controlled partially observable markov decision processes, Decision and Control (2000) 1: 124-129.

[37] A. El-Fakdi, M. Carreras, N. Palomeras, Direct policy search reinforcement learning for robot control, in: Artificial Intelligence Research and Development, 2005.

[38] A. El-Fakdi, M. Carreras, N. Palomeras, P. Ridao, Autonomous underwater vehicle control using reinforcement learning policy search methods, Oceans (2005) 2: 793-798.

[39] S. Dejmal, A. Fern, T. Nguyen, Reinforcement learning for vulnerability assessment in peer-to-peer networks, in: Innovative Applications of Artificial Intelligence, 2008.

[40] Y. Abu-Mostafa, Learning from hints in neural networks, Journal of Complexity 6 (2) (1990) 192-198. 
[41] J. Bridle, Probabilistic interpretation of feedforward classification ..., Neurocomputing: Algorithms, Architectures, and Applications (1990) $227-236$.

[42] R. Duda, P. Hart, D. Stork, Pattern Classification, John Wiley and Sons, 2006.

[43] S. Nolfi, D. Floreano, O. Miglino, F. Mondada, How to evolve autonomous robots: Different approaches in evolutionary robotics, in: Proc. of Artificial Life IV, 1994, pp. 190-197.

[44] D. Moriarty, R. Miikkulainen, Forming neural networks through efficient and adaptive coevolution, Evolutionary Computation 5 (2002) 373-399.

[45] K. Tumer, A. Agogino, Coordinating multi-rover systems: Evaluation functions for dynamic and noisy environments, in: The Genetic and Evolutionary Computation Conference, Washington, DC, 2005.

[46] A. Agogino, K. Tumer, R. Miikulainen, Efficient credit assignment through evaluation function decomposition, in: The Genetic and Evolutionary Computation Conference, Washington, DC, 2005. 